Ann. Biol. anim. Bioch. Biophys., I973, 13 (3), 347-362.

\title{
SPÉCIFICITÉ D'ACTION ET RELATIONS IMMUNOLOGIQUES DES HORMONES GONADOTROPES DE QUELQUES TÉLÉOSTÉENS
}

\author{
B. BRETON, R. BILLARD et B. JALABERT \\ avec la collaboration technique de Anne-Marie Escaffre et Marie-Claire Théron \\ Laboratoire de Physiologie des Poissons, \\ Centre national de Recherches zootechniques, I. N. R. A., \\ 78350 Jouy en Josas \\ - RÉSUMÉ
}

Il existe une confusion importante dans la notion de spécificité d'action des hormones gonadotropes de Poissons. Cette notion a été précisée en utilisant deux méthodes différentes : d'une part la comparaison des activités biologiques de différents extraits hypophysaires de Poissons sur un même récepteur testiculaire, d'autre part la détermination de leur comportement immunologique vis-à-vis d'anticorps hautement spécifiques dirigés contre I'hormone gonadotrope de Carpe $c$-HG et $o$-LH. La spermatogenèse du Carassin, Carassius auratus et du Guppy, Poecilia reticulata hypophysectomisés ne peut être regénérée quantitativement qu'avec des hormones gonadotropes ou extraits hypophysaires autologues d'une espèce donneuse très proche de l'espèce receveuse. La technique de compétition isotopique vis-à-vis d'anticorps spécifiques d'une entité moléculaire définie, donne des résultats comparables. Seuls des extraits hypophysaires de Carpe et d'autres Cyprinidés peuvent déplacer de l'hormone de Carpe marquée à l'iode ${ }^{131}$ I ( $c$-HGI $\left.{ }^{\text {131 }}\right)$ du complexe $c$-HGI ${ }^{131}$, anti $c$-HG. Les hormones mammaliennes $o-\mathrm{LH}$, o-FSH et HCG n'ont en aucun cas montré d'activité sur la spermatogenèse du Carassin et du Guppy ni d'affinité vis-à-vis d'anticorps dirigés spécifiquement contre une hormone gonadotrope de Poisson. Ces résultats confirment l'existence d'une spécificité zoologique d'action des hormones gonadotropes hypophysaires entre les Mammifères et les Poissons Téléostéens et à l'intérieur même des différentes familles de Téléostéens.

Depuis les premières observations de Houssay et al. (I929 $a, b)$ de nombreux travaux (revus par PICKFord et ATz, I957 ; HoAR, I965 ; FonTAINE et al., I966) ont suggéré l'existence d'une spécificité zoologique des hormones gonadotropes de Poissons, non seulement par rapport aux Mammifères ou aux Vertébrés inférieurs, mais au sein même des Téléostéens.

Ainsi, si l'on considère l'action d'extraits hypophysaires de Poissons sur des Vertébrés plus évolués, on constate que leurs activités gonadotropes sur des tests 
biologiques caractéristiques d'une activité LH ou FSH varient selon les espèces donneuses d'hypophyse et receveuses (revue de PICKFord et ATz, r957; OTsukA, I956, r960). BURZAWA-GERARD et FONTAINE (I965), n'ont observé aucune action d'une hormone gonadotrope purifiée de Carpe sur les tests de Parlow et de SteelmanPohley, alors que Burzawa-Gerard (I968) a mis en évidence une activité de type FSH dans 1'hypophyse du Protoptère. Seuls les tests réalisés sur Batraciens (spermiation et hydratation du testicule) ont montré une réponse positive constante aux extraits hypophysaires de Poissons (Fontaine et Chauver, I96I ; Delsol et FLATIN, Ig64). Ces tests ne sont cependant pas spécifiques d'une activité de type LH ou FSH. Enfin Licht et Donaldson (I969), Licht et STOCKELL-HARTREE (I97I) obtiennent des résultats contradictoires sur les activités respectives d'hormones gonadotropes partiellement purifiées de Saumon et de Carpe sur un même récepteur : le testicule du Lézard Anolis carolinensis hypophysectomisé.

Inversement, si certains auteurs (Ashan, I966; Sundararaj et Goswami, I966 ; Sundararaj et Nayyar, I967; Goswami et Sundararaj, I968) ont trouvé une activité des hormones gonadotropes mammaliennes LH et FSH dans certaines espèces de Poissons Téléostéens, BILLARD et al. (I970, I97I) ne retrouvent pas cette activité chez le Carassin. Outre le problème de spécificité, ces différentes données posent également celui de l'unicité ou de la dualité du facteur gonadotrope (BuRzawa-Gerard et Fontaine, I966; Burzawa-Gerard et Fontaine, I972).

En ce qui concerne l'activité des hormones gonadotropes à l'intérieur même du groupe des Poissons, on retrouve ce même concept de spécificité (PICKFord et ATz, r957). Clemmens et al. (I964), Clemmens et Grant (I964), Clemens et Johnson (I965) ont observé des réponses différentes dans le test d'hydratation du testicule du Carassin Carassius auratus suivant l'espèce donneuse d'hypophyse.

En toute rigueur, seule la restitution de séquences complètes et durables de la gamétogenèse chez des animaux hypophysectomisés peut constituer un critère d'activité gonadotrope. Ainsi, JALABERT (I969) n'observe aucune activité d'extraits hypophysaires de Gardon (Cyprinidé) sur la vitellogenèse de Poecilia reticulata hypophysectomisé (Pœcilidé) alors qu'elle est maintenue par des extraits hypophysaires de Gambusia, Poisson appartenant à la même famille.

En utilisant le test de la spermiation du Carassin, Yamazaki et Donal,dson (I968) ont pu purifier partiellement une hormone gonadotrope de Saumon. Ces auteurs n'ont malheureusement pas comparé l'activité de leur préparation, à celle d'extraits hypophysaires de Carassin sur ce test. En fait, dans leur ensemble, toutes ces contradictions apparentes quant à la " spécificité " des hormones gonadotropes de Poissons peuvent s'expliquer par l'imprécision même de ce concept : la spécificité observée expérimentalement tient autantà la nature même du test biologique choisi, plus ou moins représentatif, qu'à la nature de l'hormone testée.

Nous avons entrepris de fournir un support objectif à la notion de spécificité des hormones gonadotropes de Poissons en comparant les résultats obtenus par deux voies d'approche différentes :

- d'une part l'activité d'extraits hypophysaires sur le maintien ou la restauration de la spermatogenèse chez les mâles hypophysectomisés de deux espèces appartenant à des familles très différentes : le Guppy, Poecilia reticulata (Poecilidae), et le Carassin (Carassius auratus, (Cyprinidae) ; cette étude, contrairement à toutes 
celles réalisées à ce jour, a été faite par analyse quantitative de la spermatogenèse qui est susceptible de donner à cette notion de spécificité une définition plus précise ;

- d'autre part, l'étude de la spécificité immunologique de ces mêmes extraits par rapport à l'hormone $c$-HG d'une espèce de Poisson servant de référence, la Carpe, et par rapport aux hormones gonadotropes mammaliennes 0 -LH et o-FSH.

\section{MATÉRIEL ET MÉTHODES}

\section{A. - Spécificité biologique}

\section{Préparation des extraits hypophysaires.}

Les hypophyses des espèces suivantes ont été utilisées : Carpe (Cyprinus carpio), Carassin (Carassius auratus), Gardon (Gardonus gardonus), Hottu (Chondrostoma nasus), Tilapia (Tilapia mossambica), Truite (Salmo Trutta), Lamproie (Lampetra fluviatilis) Gambusia sp. (Gambusia affinis), Esturgeon (Acipenser guldenstädti).

Les extraits sont préparés à partir d'hypophyses fraîches ou lyophilisées congelées dans l'azote liquide aussitôt après décapitation et prélèvement. Ces prélèvements ont eu lieu pendant la période de gamétogenèse, au cours de laquelle la charge hypophysaire en hormone gonadotrope est supposée la plus forte. Les hypophyses sont broyées dans l'acétone à $-20^{\circ} \mathrm{C}$. Le broyat est agité pendant $\mathrm{I} 2$ heures dans l'acétone à $4^{\circ} \mathrm{C}$, puis filtré sur büchner. La poudre brute obtenue est lavée deux fois sur büchner au $\mathrm{N}$ butanol et une fois à l'éther, afin d'obtenir une délipidation totale.

\section{Supplémentation sur Poecilia reticulata (maintien de la spermatogenèse).}

Les conditions d'élevage (BILLARD, I969a), la technique d'hypophysectomie (JALABERT et BILIARD, 1968) et la méthode d'analyse quantitative de la spermatogenèse (BILLARD, I969 $b$ ) ont déjà été décrites. Le contrôle de l'hypophysectomie est assuré par histologie à la fin de l'expérience.

Les préparations hypophysaires de Gambusie, Carpe, Gardon et Hottu sont administrées par voie intrapéritonéale (à la base du gonopode), grâce à une pompe à microinjection (Beaudoin) équipée d'une microaiguille de quartz préalablement étalonnée et affútée en biseau ; du fait du faible diamètre de l'aiguille les préparations acétoniques hypophysaires ne peuvent être injectées à l'état de suspensions brutes et subissent l'extraction suivante : après mélange de la poudre hypophysaire à raison de $\mathrm{I} \mathrm{mg}$ pour $\mathrm{o,2} \mathrm{ml}$ de solvant (solution saline de $\mathrm{NaCl}$ à $0,15 \mathrm{M}$ tamponnée à $\mathrm{pH} 7,0$ ), la préparation subit une série de congélations et de décongélations, un broyage au "potter ", puis une centrifugation de $5 \mathrm{mn}$ à 3 ooo $\mathrm{tr} / \mathrm{mn}$. Les animaux mâles, d'un poids moyen de $100 \mathrm{mg}$ reçoivent $2 \mu \mathrm{l}$ de surnageant, contenant l'équivalent de $10 \mu \mathrm{g}$ de poudre initiale. Les injections sont faites tous les jours pendant 18 jours et débutent 1 jour après l'hypophysectomie.

Les hormones mammaliennes (o-LH-CNRS. $\mathrm{M}_{2}=1,94$ LH-NIHS,$o$-FSH-CNRS. H65-206 $=2.03$ FSH NIH $S_{2}$ et HCG-Organon) ont été administrées aux doses respectives de $5 \mu \mathrm{g}$ et 5 UI/animal, au même rythme que précédemment. Elles sont dissoutes dans le même solvant auquel on ajoute r p. Iooo de gélatine. Des supplémentations réalisées avec l'hormone purifiée $c$-HG à la dose de I $\mu \mathrm{g} / \mathrm{mâle}$ et par injection n'ont pu donner lieu à une exploitation quantitative de la spermatogenèse du fait d'une forte mortalité accidentelle survenue dans ce groupe et seul le RGS a été calculé.

\section{Supplémentation sur Carassius auratus.}

Après hypophysectomie les Carassins d'un poids de 30 à $50 \mathrm{~g}$ sont placés par lots de 3 ou 4 animaux dans des aquariums de 201 d'eau salée (sel de cuisine à 8 p. r ooo), oxygénée par diffusion d'air comprimé et maintenus à $17^{\circ} \mathrm{C}$ sous une photopériode de 12 L-I $2 \mathrm{~N}$.

L'hypophysectomie est pratiquée par voie operculaire selon la technique de Chavin (I956), modifiée par LAHLOU et SAWYER (1969).

Le contrôle histologique de l'hypophysectomie est réalisé a posteriori après fixation de la selle turcique et de la base de l'hypothalamus au Bouin-Hollande et coloration au Bleu Alcian (pH r,5)-PAS-Orange G. Dans certains cas ce contrôle a été complété par des tests d'immunofluorescence (BILLARD et al., 1971). 
Les préparations hypophysaires et les hormones gonadotropes sont administrées en injections intrapéritonéales de $20 \mu \mathrm{l}$, au rythme de $3 /$ semaine pendant 24 jours à des doses variant de $0, \mathbf{I}$ à $100 \mu \mathrm{g} /$ injection/gramme de poids corporel. Les préparations acétoniques hypophysaires sont administrées sous forme de suspension après broyage dans du sérum physiologique à 8 p. 1 ooo.

Les supplémentations sont réalisées dans les conditions suivantes :

a) Expériences de maintien de la spermatogenèse.

Le traitement débute 2 ou 3 jours après l'hypophysectomie et dure 3 semaines à raison de 3 injections/semaine, avec les préparations suivantes : poudres hypophysaires acétoniques de Carpe et de Gardon, hormones gonadotropes de Carpe : $c$-HG (BURzaWA-Gerard, I97I) et de Mammifères : LH, FSH et HCG-Organon, déjà mentionnées à propos des supplémentations sur Poecilia.

b) Expériences de vegénération de la spermatogenèse.

Le traitement de supplémentation débute seulement $3^{\circ}$ jours après l'hypophysectomie, lorsque les spermatozoïdes sont en grande partie résorbés dans le testicule. Il est poursuivi pendant 4 semaines à raison de 3 injections/semaine avec des préparations acétoniques d'hypophyse de Carpe, de Truite et de Tilapia, et les mêmes hormones mammaliennes que dans l'expérience de maintien de la spermatogenèse. Les animaux témoins sont constitués d'une part par les animaux hypophysectomisés ne recevant que le solvant et d'autre part par des mâles intacts élevés dans les mêmes conditions de température et de photopériode que les animaux expérimentaux.

Dans les 2 cas la spermatogenèse avait déjà commencé lors de l'hypophysectomie.

\section{B. - Spécificité immunologique}

Trois types de techniques ont été utilisés : l'immunoélectrophorèse, l'étude de la compétition des extraits hypophysaires vis-à-vis de deux systèmes radioimmunologiques de référence $c$-HG et o-LH, et l'étude de la liaison de l'hormone gonadotrope de Carpe $c$-HG à des anticorps anti $c-\mathrm{HG}, o-\mathrm{LH}$ et $o-\mathrm{FSH}$.

\section{Préparation des extraits hypophysaires.}

Les extraits hypophysaires sont préparés à partir des poudres acétoniques soumises à une extraction de $\mathrm{I} 2$ heures à $4^{\circ} \mathrm{C}$ dans un tampon véronal $0,025 \mathrm{M}$ à pH 8,6 contenant $2,5 \mathrm{p}$. I ooo de sérum albumine. L'extraction est réalisée dans le rapport quantitatif initial de I $\mathrm{mg}$ de poudre acétonique brute/ml de milieu d'extraction. L'extrait final est fourni par le surnageant après centrifugation de $20 \mathrm{mn}$. à 3 ooo $\mathrm{tr} / \mathrm{mn}$.

\section{Immunoélectrophorèses.}

Elles ont été réalisées sur plaques de $25 \times 76 \mathrm{~mm}$ en gel d'Ionagar (Difco) à I p. roo de I $\mathrm{mm}$ d'épaisseur. La migration s'effectue sous une tension de $6 \mathrm{~V} / \mathrm{cm}$ pendant 3 heures, l'antigène est déposé à la concentration de $\mathrm{I} \mathrm{mg} / \mathrm{ml}$ sous un volume de Io $\mu \mathrm{l}$. Après immunodiffusion de 48 heures, les lames sont lavées 24 heures à l'eau distillée, puis 24 heures avec une solution de $\mathrm{NaCl} \mathrm{N}$ avant d'être colorées par l'amido-Schwartz à I p. I ooo dans l'acide acétique à $7 \mathrm{p}$. Ioo.

3. Étude de la compétition entre c-HG, o-LH et des extraits hypophysaires de Poissons vis-à-nis de deux systèmes radioimmunologiques de référence.

Les conditions d'utilisation de ces deux systèmes $c$-HG et $o$-LH ont été précédemment décrites (BRETON et al. (I97I). Les deux hormones sont marquées par 1'131I selon la technique de GREENWOOD et al. (1963). Les anticorps préparés sur Cobayes sont utilisés à la concentration de $\mathrm{I} / 330$ ooo pour l'anti $c-\mathrm{HG}$ et $\mathrm{I} / 380$ ooo pour l'anti $o$-LH. Les résultats sont obtenus après 4 jours d'incubation à $4^{\circ} \mathrm{C}$ en tampon véronal o,o25 $\mathrm{M}$ à $\mathrm{pH} 8,6$ contenant $2,5 \mathrm{p}$. I ooo de sérum albumine humaine. L'hormone libre est séparée de l'hormone liée à l'anticorps par immunoprécipitation du complexe par du sérum de Lapin anti $\gamma$-globulines de Cobaye.

Les résultats sont exprimés par la décroissance de radioactivité :

$$
\frac{\text { hormone liée à l'anticorps }}{\text { hormone libre }}=\frac{\mathrm{B}}{\mathrm{F}}
$$

en fonction de la quantité d'hormone froide, ou d'extrait hypophysaire. Les extraits hypophysaires suivants ont été utilisés : Carpe, Carassin, Gardon, Truite, Tilapia, Lamproie, Esturgeon, Gambusie, Mouton, ainsi que les hormones ovines LH et FSH, et HCG. 
4. Étude de la liaison de c-HG et o-LH à des anticorps antihormones gonadotropes (titrage des anticorps).

L'étude a porté sur le pourcentage de liaison de $c-\mathrm{HG}^{131}$ à des anticorps anti $c-\mathrm{HG}, o-\mathrm{LH}$, $o-\mathrm{FSH}$ et $o-\mathrm{LH}^{131} \mathrm{I}$ à des anticorps anti $o-\mathrm{LH}$ et $c-\mathrm{HG}$, en fonction de la concentration en anticorps.

\section{RÉSULTATS}

\section{A. - Etude biologique}

\section{Supplémentation sur Poecilia (tabl. I).}

Les préparations hypophysaires brutes de Gambusia maintiennent de façon satisfaisante la spermatogenèse du Guppy hypophysectomisé. Les stades les plus sensibles à 1'hypophysectomie (dernières générations spermatogoniales, spermatocytes $I$ et premières phases de la spermiogenèse) ne sont que partiellement maintenus mais les valeurs observées restent très supérieures à celles des animaux témoins hypophysectomisés. Les préparations hypophysaires des autres espèces de Poissons étudiées, sont beaucoup moins actives ; celles dela Carpe et du Hottu semblent avoir moins d'effet que celles du Gardon avec laquelle la quantité de spermatocytes I reste légèrement plus élevée.

Après les supplémentations réalisées avec l'hormone gonadotrope de Carpe hautement purifiée ( $c$-HG) le RGS moyen des Guppy traités, établi à partir de 4 animaux est de $I, 65$ (extrèmes $I, 45$ et $I, 82$ ). Parmi les hormones mammaliennes, seule HCG maintient partiellement le RGS mais 1'analyse histologique montre que la quantité de cellules germinales est faible et que c'est la prolifération de tissu conjonctif qui est responsable des valeurs élevées du RGS. Les premières générations spermatogoniales $\mathrm{G}_{1}-\mathrm{G}_{7}$ sont mieux maintenues par $0-\mathrm{FSH}$ que par $0-\mathrm{L} H$. Il faut égale-

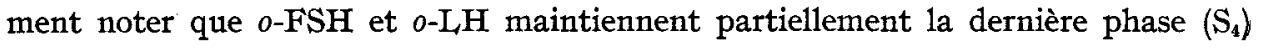
de la spermiogénèse.

\section{Supplémentation sur Carassin.}

a) Expériences de maintien (tab1. 2).

A la dose de I $\mu \mathrm{g}$, la poudre brute d'hypophyses de Carpe maintient la spermatogenèse au niveau de celle des témoins comme l'hormone $c-H G$ à $o, I \mu g$; à la dose de Io $\mu \mathrm{g}$ la spermatogenèse est fortement stimulée. Par contre, la poudre hypophysaire de Gardon ne maintient que partiellement les spermatogonies B, les spermatocytes I et les spermatozoïdes des lobules testiculaires.

b) Expérience de regénération (tabl. 3).

Seul 1'extrait hypophysaire de Carpe est capable de regénérer la spermatogenèse du Carassin hypophysectomisé à un niveau comparable à celui des témoins intacts. Avec les extraits hypophysaires de Truite la spermatogenèse n'est restaurée que de façon qualitative avec un rendement apparemment très faible même à la dose $100 \mu \mathrm{g} / \mathrm{g}$ de poids vif. Le niveau de restauration est encore plus faible avec les préparations hypophysaires de Tilapia : seules les dernières générations spermatogoniales se 










reconstituent et la méiose n'est jamais engagée. Les hormones mammaliennes n'ont aucun effet et le RGS est particulièrement faible, excepté pour HCG qui maintient mieux la structure des cellules de Sertoli que LH et FSH.

3. Étude du déplacement de $\mathrm{c}-H G{ }^{131} I$ et $\mathrm{o}-L H{ }^{131} I$, de leurs complexes antigène-anticorps par des extraits hypophysaires de Poissons et des hormones mammaliennes.

Les extraits hypophysaires de Carpe et de Carassin inhibent totalement la liaison de $c$-HG ${ }^{131}$ I à son anticorps homologue (fig. I). Par contre, cette liaison n'est pas totalement inhibée par l'extrait hypophysaire d'un autre Cyprinidé, le Gardon.

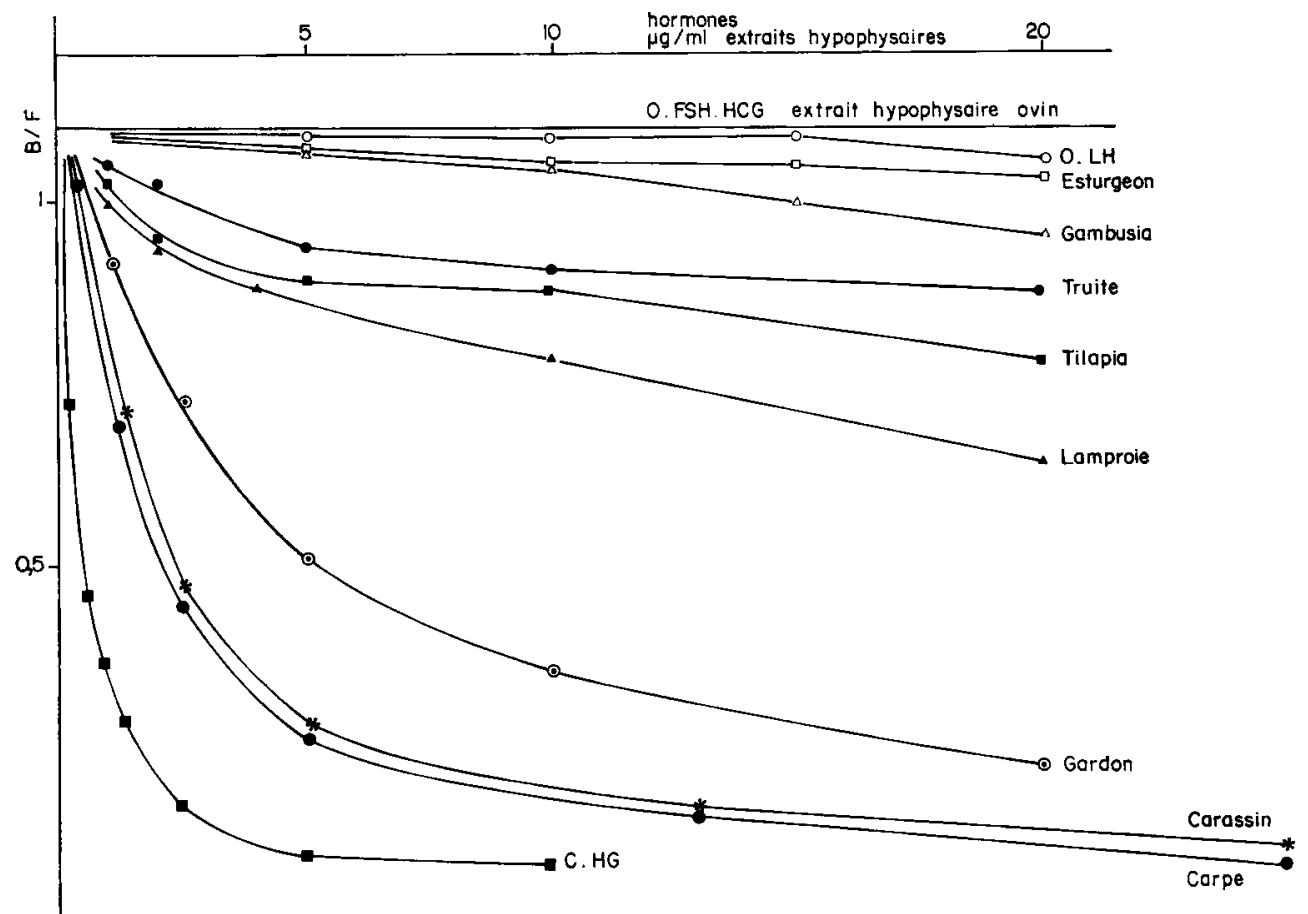

$102050 \quad 100$

Fig I. - Courbes de deplacement de c-HG ${ }^{131} I$ du complexe c- $H G{ }^{131} I$ anti c-HG par différents extraits hypophysaires de Poissons

it des hormones gonadotropes mammaliennes

Dans les deux cas il y a peu de différence dans les pourcentages d'inhibition obtenus, mais les concentrations d'extraits hypophysaires nécessaires pour obtenir des résultats équivalents varient de 0,025 à $0.5 \mu \mathrm{g} / \mathrm{ml}$ dans le cas de la Carpe et du Carassin, et de I à $20 \mu \mathrm{g} / \mathrm{ml}$ pour le Gardon. Les autres extraits hypophysaires utilisés (Truite, Tilapia) ne déplacent que très partiellement $c$-HG ${ }^{181} \mathrm{I}$ du système $c$-HG ${ }^{181}$ I-anti $c-\mathrm{HG}$; ce résultat indique une chute de liaison immunologique entre l'anticorps anti-hormone gonadotrope de Carpe et les principes gonadotropes contenus dans ces extraits hypophysaires. Cette liaison devient très faible ou. nulle avec des extraits provenant de Gambusia et Esturgeon ou des hormones gonadotropes mammaliennes $o$-I,H, o-FSH et HCG. 
De même (fig. 2) seul l'extrait de poudre acétonique d'hypophyse de Mouton est susceptible d'inhiber totalement la liaison de $o-\mathrm{L} H{ }^{131} \mathrm{I}$ à l'anti $o-\mathrm{L} / \mathrm{H}$. Les extraits hypophysaires de Poissons ne présentent que peu ou pas d'affinité vis-à-vis de ce système.

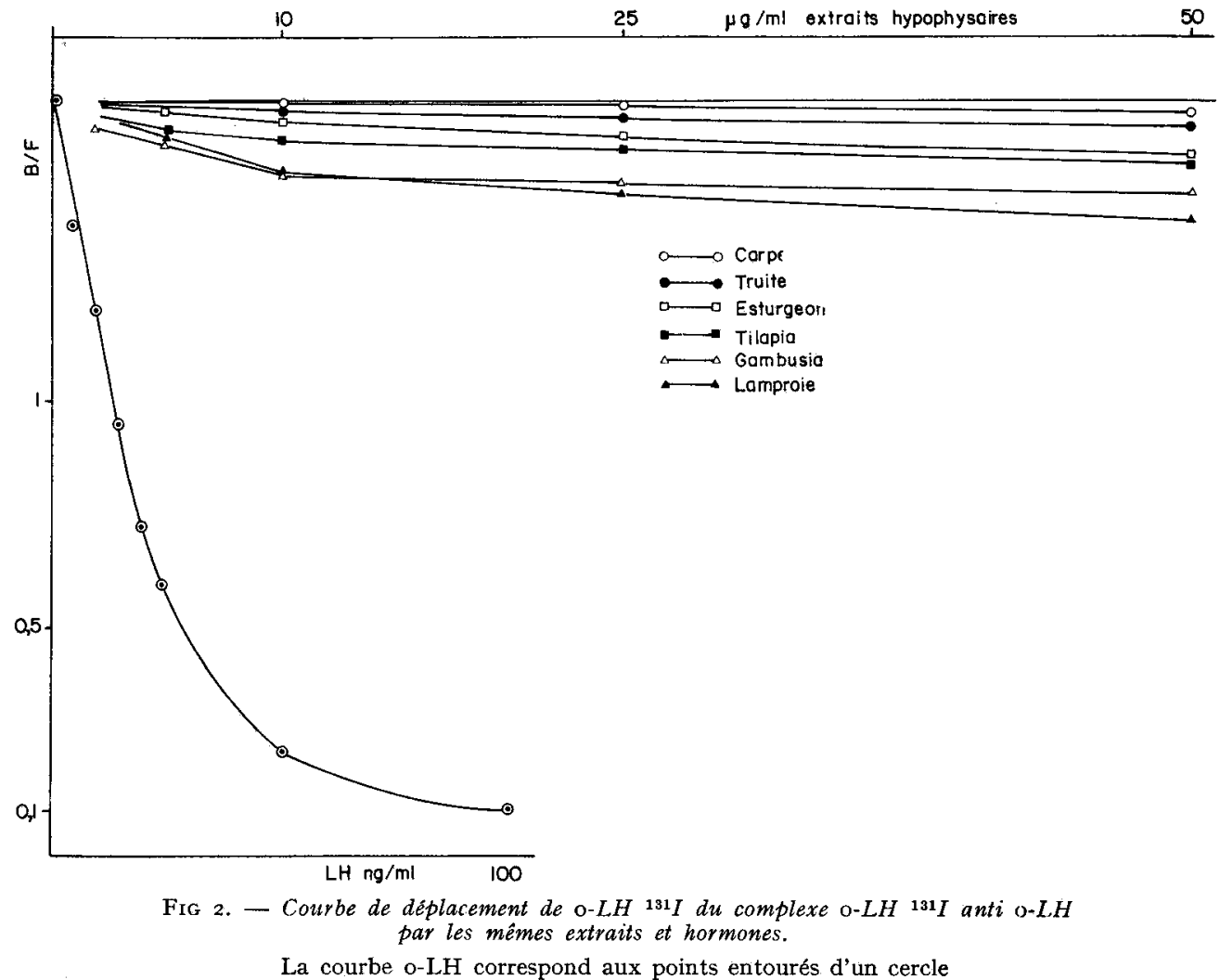

Dans les deux systèmes la plupart des extraits hypophysaires donnent des courbes de liaison qui ne sont pas parallèles aux courbes de référence, à l'exception des extraits de Carpe, Carassin et Gardon, vis-à-vis du système $c$-HG et de l'extrait hypophysaire ovin vis-à-vis du système $o-\mathbf{L} H$.

4. Étude des liaisons de c-HG et o- $L H$ à des anticorps anti-hormone gonadotrope (fig. 3 et 4 ).

L'hormone marquée $c-H G{ }^{131}$ I ne se lie bien qu'à l'anticorps anti $c-H G$. Elle ne montre pas de liaison vis-à-vis de l'anti o-FSG et environ 5 p. Ioo de liaison vis-à-vis de 1'anti $o$-LH (fig. 3 ).

De même, la $o-\mathrm{L} H{ }^{13 I}$ I ne présente qu'une faible affinité pour l'anticorps anti c-HG (fig. 4).

Les immunoélecţrophorèses des extraits hypophysaires de Poissons autres que la Carpe avec l'anti-gonadotrope de Carpe n'ont pas donné de réaction positive. Avec l'extrait hypophysaire de Gardon on note cependant un faible arc de précipitation. 


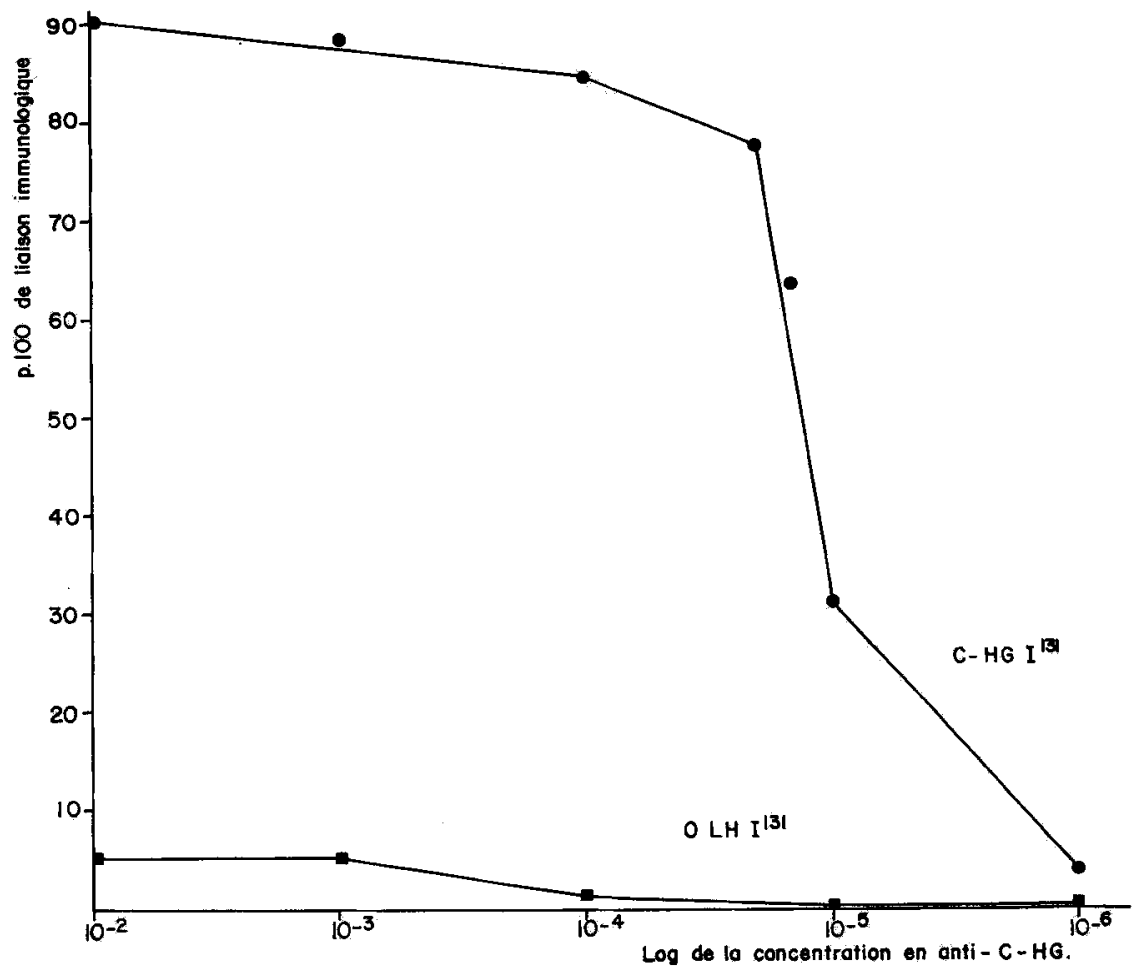

Fic. 3. - Liaison de o- $H G$ et o- $L H$ a l'anticorps anti c- $H G$

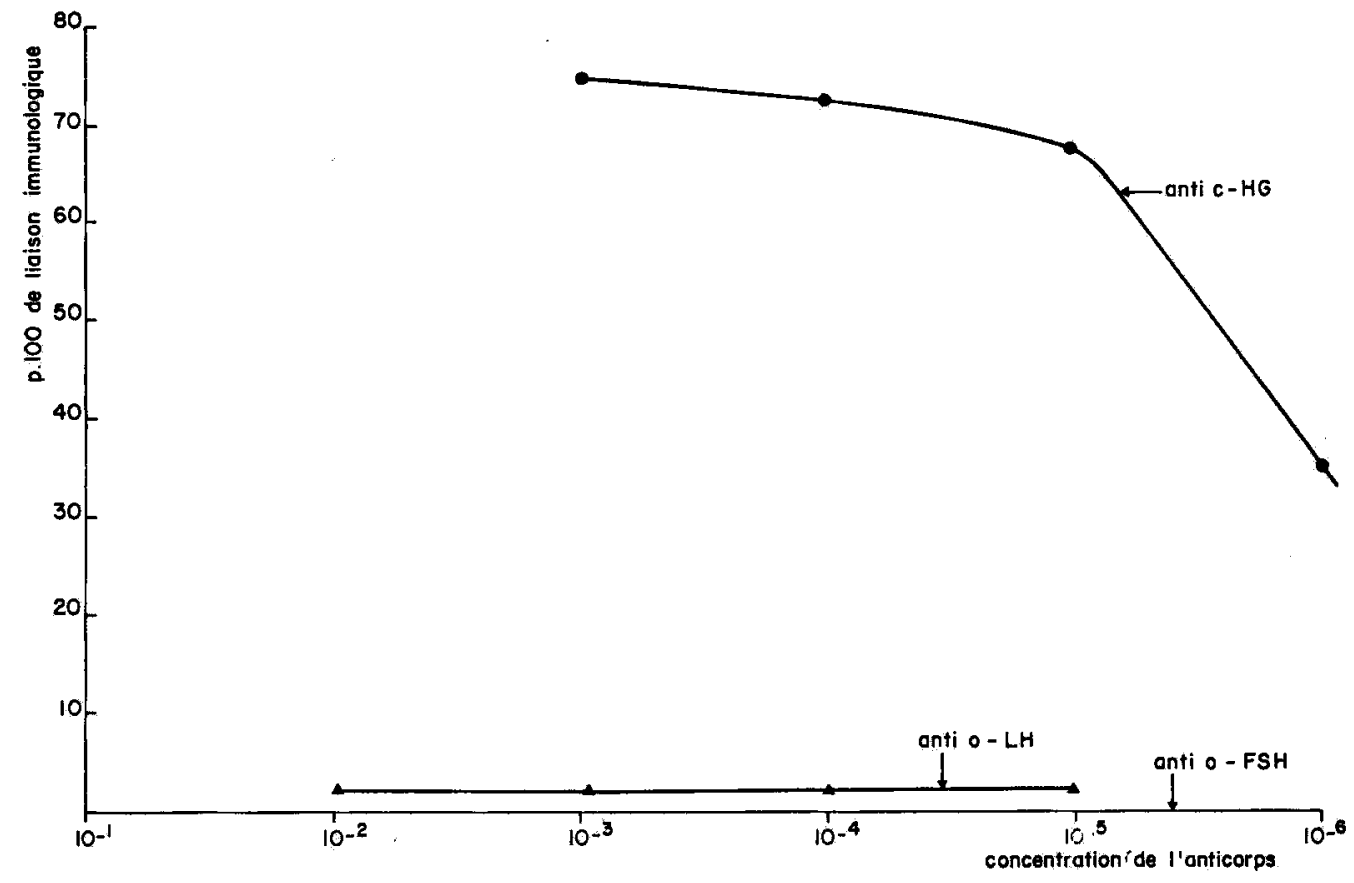

FIG. 4. - Liaison de c-HG ${ }^{131} I$ dे des anticorps anti $0-L H, 0-F S H$ et c-HG 


\section{DISCUSSION}

Les résultats obtenus sur le maintien ou la restauration de la spermatogenèse de Poecilia et Carassius font apparaître des différences considérables dans les effets des préparations hypophysaires testées.

Des doses de $c$-HG de $0, \mathrm{I} \mu \mathrm{g} / \mathrm{g}$ administrées tous les 2 ou 3 jours, maintiennent la spermatogenèse du Carassin alors que des doses quotidiennes roo fois plus élevées n'ont pas d'effet chez le Guppy. Parmi les préparations hypophysaires brutes, seule celle de Gambusia maintient la spermatogenèse de Poecilia hypophysectomisé et celle de Carpe assure le maintien et la regénération de la gamétogenèse du Carassin privé d'hypophyse, ce qui confirme les résultats antérieurs de BILLARD et al. (I97o, I97I) ; dans les deux cas il s'agit de couples d'espèces appartenant aux mêmes familles. Plus généralement, l'effet des extraits hypophysaires bruts, déjà variable lorsque donneurs d'hypophyses et receveurs, d'espèces différentes, appartiennent à la même famille (par exemple Carpe sur Carassin, très voisins d'une part, et Gardon ou Hottu sur Carassin d'autre part), est beaucoup plus faible lorsque donneurs et receveurs ne sont pas de la même famille. A la limite, lorsque donneurs et receveurs n'appartiennent même pas à la même classe, l'effet observé est nul : c'est le cas des hormones gonadotropes mammaliennes $o-\mathrm{L} H, o-\mathrm{FSH}$ et $\mathrm{HCG}$ sur Carassin et $o-\mathrm{LH}$ et 0 -FSH sur Poecilia. Il n'est toutefois pas exclu que des doses plus élevées aient un effet positif. Cependant, en considérant le critère du maintien ou de la regénération de la spermatogenèse, par des doses équivalentes des différents extraits, il est possible de conclure à l'existence d'une spécificité zoologique dans l'action des hormones gonadotropes à l'intérieur même du groupe des Téléostéens. Cette notion de spécificité zoologique est qualitative et 1'on doit admettre qu'elle peut être compensée, dans une certaine mesure, par 1'administration de doses plus élevées, voire extraphysiologiques, permettant d'obtenir sensiblement le même effet qu'une très faible dose de l'hormone gonadotrope homologue. Ces considérations permettent de comprendre les contradictions apparentes entre nos résultats et d'autres travaux récents : c'est ainsi que YAMAZAKI et DoNALDSON (I968) ont regénéré la spermatogenèse du Carassin hypophysectomisé avec des extraits hypophysaires partiellement purifiés de Saumon SG-G ${ }_{100}$, mais l'explication réside probablement dans les différences de doses et d'activité des préparations utilisées. D'après le test de maturation in vitro des ovocytes de la Truite, $\mathrm{SG}-\mathrm{G}_{100}$ est 60 fois plus actif que la préparation brute de Truite dont nous disposons (JALABERT et al., I973). Compte tenu des doses administrées par YAMAZAKI et DONALDSON, les quantités d'hormones mises en œuvre étaient 60 fois plus élevées que dans la présente expérience. Il est dans ces conditions vraisemblable que de telles doses extraphysiologiques, aient pu régénérer au moins partiellement la spermatogenèse à un niveau que l'absence d'évaluation quantitative ne permet pas d'apprécier.

En ce qui concerne les regénérations de la spermatogenèse et les stimulations ovariennes du Poisson-Chat indien Heteropneustes fossilis, observées par SunDaRARAJ et ses collaborateurs (Sundararaj et Goswami, I966 ; SundararaJ et Nayyar I967, SUNDARARAJ et al., I972 ; SUNDARARAJ et ANAND, 1972), elles sont vraisem- 
blablement le résultat d'une compensation de spécificité par surdosage. Il est d'ailleurs apparu récemment que $\mathrm{L}_{1} H$ considérée comme gamétocinétique dans cette espèce était cependant Io fois moins active que SG-G 100 $_{\text {(SUNDARARAJ et ANAND, }}$ 1972).

Dans un travail récent, FonTAINE et al. (r972) considérant 1'effet de 2 hormones gonadotropes pisciaires hautement purifiées (Carpe $=c$-HG, BURZAWA-GERARD, I97I ; et Saumon, Donal,Dson et al, I972) sur un même récepteur (activité adenylcyclasique de l'ovaire de Carassius auratus) concluent aussi à l'existence d'une spécificité d'action de ces deux hormones.

Les résultats obtenus par immunologie recoupent les précédents. Les déplacements observés montrent qu'il existe à l'intérieur même du groupe des Téléostéens une spécificité immunologique importante entre les hormones gonadotropes. L,es 3 espèces de Cyprinidés testés : Carpe, Carassin, Gardon, ne montrent pas de différences antigéniques importantes alors que le croisement immunologique diminue plus ou moins par rapport à la référence Carpe, avec les autres espèces utilisées, selon les familles auxquelles elles appartiennent. Ces résultats suggèrent qu'il doit exister des déterminants antigéniques communs à certaines hormones gonadotropes de Poissons, et une évolution de la molécule hormonale selon les espèces, certaines (Gambusia, par exemple) ne possédant aucun caractère immunologique de 1'hormone utilisée dans le système de référence.

L'absence de réactions immunologiques entre la LH, la FSH, 1'HCG et 1'anti $c$-HG d'une part, et $c$-HG et les anti $o-\mathrm{LH}, o-\mathrm{FSH}$ d'autre part, montre qu'il ne doit pas $\mathrm{y}$ avoir de communauté antigénique entre ces hormones mammaliennes et l'hormone gonadotrope de Carpe. L'analyse séquentielle de cette hormone n'a pas été réalisée, mais sa composition en résidus d'acides aminés par moles (BURzAWAGERARD, I968) est très différente de celle de LH, de FSH de différentes origines : ovine (DE LA LIOSSA et al., I968) ; PAPKOFF et al., I967) ; bovine (LIAO et PIERCE, I970) ; humaine (SAXENA et RATHNAM, I97I) et de celle de HCG.

Les faits expérimentaux exposés dans cette étude immunologique et biologique, ne semblent pas confirmer que l'hormone gonadotrope de la Carpe et du Carassin soit de type LH, FSH ou HCG. Cette hormone gonadotrope serait également différente de la L,H de Poulet ainsi que le démontrent les résultats de ScaNEs et al. (I972). Avec un anticorps anti- $\mathrm{LH}_{\mathrm{H}}$ de Poulet, des réactions croisées complètes ne peuvent être obtenues qu'avec des extraits hypophysaires d'autres oiseaux. Les hormones purifiées 0 -LH, o-FSH, les hormones gonadotropes de Tortue (PAPKOFF et LICHT, I972) et de Carpe ne montrent qu'une liaison équivalente à celle de la $0-\mathrm{L} H$ à l'anti $c$-HG. Inversement de la $\mathrm{LH}$ de Poulet $\mathrm{IRC}_{2}{ }^{\left({ }^{1}\right)}$ n'a pas montré de liaison avec l'anti $c-\mathrm{HG}$ utilisé dans cette expérience.

La $c$-HG serait une hormone propre aux Cyprinidés, sans similitude avec celles des Mammifères et celles des Vertébrés des Classes supérieures.

Reçu pour publication en avril 1973.

(1) Cette LH nous a été fournie par le Dr. B. K. Foller que nous remercions. 


\section{REMERCIEMEN'TS}

Les auteurs remercient Mme BURzawa-GERARD qui nous a aimablement fournie l'hormone $c$-HG, ainsi que Mme A. S. GinsBurg (Institute of Developmental Biology, Moscou) pour l'envoi des hypophyses d'Esturgeon utilisées dans cette étude. Nous sommes également reconnaissants à MM. LAMARgue et CuINAT, Station d'Hydrobiologie à Biarritz, pour la fourniture des Gambusies et Lamproies sur lesquels les hypophyses ont été prélevées. Les hormones mammaliennes $\mathrm{LH} \mathrm{M}_{2}$ et FSH H65-206 nous ont aimablement été fournies par le Laboratoire des Hormones polypeptidiques du C. N. R. S. 78 - Gif/Yvette.

Nous remercions également l'Institut national de la Recherche agronomique et la D. G.R. S. T. (contrat $n^{\circ} 7$ I. $7 \cdot 3025$ ) qui ont supporté ce travail.

\section{SUMMARY}

\section{ACTION SPECIFICITY AND IMMUNOLOGICAL, RELATIONS OF GONADOTROPIC HORMONES IN SOME TELEOST FISH}

There is widespread confusion in the idea of action specificity of gonadotropic hormones in fish. Two methods were used to define this notion : comparison of biological activities of various pituitary extracts of fish on the same testicular receiver ; determination of their immunological behavior in relation to highly specific antibodies against carp gonadotropic hormone, $c$-HG and o-LH. Spermatogenesis of hypophysectomized goldfish, Carassius auratus, and guppy, Poecilia reticulata, can only be quantitatively regenerated with gonadotropic hormones or autologous pituitary extracts of a donnor species very similar to the receiver species. The isotopic competition technique gives comparable results in relation to specific antibodies having a definite molecular entity. Only pituitary extracts of carp and other cyprinids can displace carp hormone labelled with iodine ${ }^{131}$ ( $(c-131 \mathrm{HGI})$ from the anti $c$-HG- $c$-HGI ${ }^{131}$ complex. Mammalian hormones $o-\mathrm{LH}, o-\mathrm{FSH}$ and HCG have never shown effect on gold fish or guppy spermatogenesis or affinity in regard to antibodies specifically against a fish gonadotropic hormone. These results confirm that pituitary gonadotropic hormones have a zoologic action specificity between mammals and Teleost fishes and even within different families of Teleosts.

\section{RÉFÉRENCES BIBLIOGRAPHIQUES}

AHSAN N. S., 1966. Effects of gonadotropic hormones on male hypophysectomized lake chub Couescius plumbeus. Canad. J. Zool., 44, 703-718.

Billard R., I969a. Hypophysectomie et spermatogenèse chez Poecilia reticulata. C. R. Acad. Sci., 268, I856-I859.

Billard R., r $969 b$. La spermatogenèse de Poecilia reticulata. I. Estimation du nombre de générations goniales et rendement de la spermatogenèse. Ann. Biol. anim. Bioch. Biophys., 9, 25I-27I.

Billard R., Burzawa-Gerard E., Breton B., i97o. Regénération de la spermatogenèse du Cyprin hypophysectomisé Carassius auratus L. par un facteur gonadotrope hautement purifié de Carpe. $C$. $R$. Acad. Sci., 272, $981-983$.

Billard R., Breton B., Dubois M. P., I971. Immunocytologie et histochimie des cellules gonadotropes et thyréotropes hypophysaires chez la Carpe Cyprinus Carpio L. C. R. Acad. Sci., 272, 1515I517.

Billard R., Breton B., Escaffre A. M., I97I. Maintien et restauration de la spermatogenèse par un extrait acétonique hypophysaire de Carpe chez le Cyprin (Carassius auratus) traité au méthallibure. Ann. Biol. anim. Bioch. Biophys., 11, 167-174.

Billard R., Solari A., Escaffre A. M., 1973. Méthodes d'analyse quantitative de la spermatogenèse de Poissons Téléostéens. Ann. Biol. anim. Bioch. Biophys. (soumis pour publication). 
Breton B,, Kann G., Burzawa-Gerard E., Billard R., i97i. Dosage radioimmunologique d'une hormone gonadotrope de Carpe Cyprinus Carpio L. C. R. Acad. Sci., 272, 981-983.

Burzawa-Gerard E., Fontaine Y. A., I 965 . Activités biologiques d'un facteur hypophysaire gonadotrope purifié de Poisson Téléostéen. Gen. Comp. Endocrinol., 5, 87-95.

Burzawa-Gerard E., Fontaine Y. A., I966. Sur le problème de l'unicité ou de la dualité de l'hormone gonadotrope hypophysaire d'un Téléostéen, la Carpe. Étude du poids moléculaire de la ou des substances actives. Ann. Endocrinol., 27, 305-310.

BurzaWa-GrRard E., 1968. Quelques propriétés des hormones gonadotropes de Poissons comparées à celles des Mammifères dans : La spécificité zoologique des hormones hypophysaires et de leurs activités, colloque C. N. R. S., Paris, I968, 351-356.

Burzawa-Gerard, I97I. Purification d'une hormone gonadotrope hypophysaire de Poisson Téléostéen, la Carpe (Cyprinus carpio L.). Biochimie, 53, 545-552.

Burzawa-Gerard E., Fontaine Y.A., 1972. Vertebrates reproduction Part. II. : The gonadotropins of lower vertebrates. Gen. Comp. Endocrinol., suppl. 3, 328-338.

Burzawa-GERARD E., I972. L'hormone gonadotrope d'un poisson téléostéen, la Carpe (Cyprinus carpio). Étude biochimique. Hormones glycoprotéiques hypophysaires. Colloque Inserm. Paris, I 5-16 mai I972, p. 2I3-222.

Chavin W., r956. Pituitary adrenal control of melanization in Xanthic goldfish Carassius auratus L. J. Exp. Zool., 133, I-36.

Clemens H. P., Grant F. B., I964 Gonadal hydratation of Carp and goldfish after injections of pituitary extracts. Zoologica, 49, 193-210.

Clemens H. P., Ciereszko L. S., Shoemaker J. D., Grant F. B., I964 Partial characterization of the gonadal hydration principle in pituitary of Carp. Gen. Comp. Endocr., 4, 503-507.

Clemens H. P., Johnson W. W. J., 1965. Specificity of the gonadal hydratation factor in the pituitary of some fresh water fishes. Copeia, 3, 389-398.

De la Liossa P., Courte C., Jutisz M., ig68. Hormone lutéinisante (LH) de Mouton. II. Étude de la composition et des groupes terminaux. Gen. Comp. Endocrinol., 11, 575-579.

Delsol. M., Flatin J., I964. Réaction du testicule du Tétard d'Alytes aux hormones gonadotropes : test de dosage FSH. Ann. Endocrinol., 25, 285-305.

Donaldson E. W., Yamazaki F., H. M. Dye, W. W. Philleo, 1972. Preparation of gonadotropin from Salmon (Oncorhynchus tshaweytscha) Pituitary glands. Gen. Comp. Endocrinol., 18, 469-481.

Fontaine M., Chauvel E., I96r. Évaluation du pouvoir gonadotrope de l'hypophyse de Poissons Téléostéens et en particulier Salmo salar L. à diverses étapes de son développement et de sa migration. C. R. Acad. Sci., 252, 822-825.

Fontaine M., Burzawa-Gerard E., Fontaine Y. A., ig66. Spécificité zoologique des hormones gonadotropes, intérêt en recherche fondamentale et appliquée. Bull. Acad. nat. Méd., 150, 45-49.

Fontaine Y.A., I969. La spécificité zoologique des protéines hypophysaires capables de stimuler la thyroïde, Acta. Endocr., suppl. 136, I-154.

Fontaine Y. A., Salmon C., Fontaine-Bertrand E., Burzawa-Gerard E., Donaldson E. W., 1972. Comparison of the activities of two purified fish gonadotropins on adenyl cyclase activity in the goldfish ovary. Can. J. Zool., 50, 1673-1676.

Goswami V.S., Sundadaraj B. I., I 668 . Effects of oestradiol benzoate HCG-FSH on unilateral ovariectomy. Induced compensatory hypertrophy in Catfish, Heteropneustes fossilis. Gen. Comp. Endocrinol., 11, 393-400.

Greenwood F., Hunter W., Glover J., I963. The preparation of ${ }^{131}$ I labeled human growth hormone of high specific radioactivity. Biochem. J., 89, II4-I23.

HoAR W. S., I965. Comparative physiology : Hormones and reproduction in fishes. Ann. Rev. Physiol., 27, $5 \mathrm{I}-7 \mathrm{O}$.

Houssay B. A. L., Lascano-Gonzales Guisti et J. M., rg29a. Implantaccion de la hipofisis y estimulacion sexual en el sapo. Rev. Soc. argent. Biol., 5, 397-418.

Houssay B. A. L., Lascano-Gonzales Guisti et J. M., I929 b. Implantation d'hypophyse et stimulation des glandes et des fonctions sexuelles du Crapaud. C. R. Soc. Biol. Paris, 162, 864-866.

Jalabert B., Billard R., r968. Hypophysectomie de Poecilia reticulata (Poisson Téléostéen). Ann. Biol. anim. Bioch. Biophys., 8, 99-150.

Jalabert B., I969. Réponse de l'ovaire de Poecilia veticulata (Poisson Téléostéen vivipare) normal ou hypophysectomisé à des injections d'extraits hypophysaires bruts de Gardon et de Gambusie. Ann. Biol. anim. Bioch. Biophys., 9, 315-328.

Jalabert B., Breton B., Billard R., I973. Dosage biologique des hormones gonadotropes de Poissons par le test de maturation in vitro des ovocytes de Truite (en préparation).

LAHLOU B., SAWYER W., I969. Electrolyte balance in hypophysectomized Goldfish Carassius auratus L. Gen. Comp. Endocrinol., 12, 370-377.

Liao T. H., PIERCE J. D., 1970. The presence of a common type of subunit in bovine thyroid stimu lating and luteinizing hormones. J. Biol. Chem., 245, 3 275-3 284.

Licht P., Donaldson E. M., 1969. Gonadotropic activity of Salmon pituitary extracts in the male Lizard (Anolis carolinensis). Gen. Comp. Endocrinol., 1, 307-314. 
Licht P., Stockell-Hartree A., 1971. Action of mammalian avian and pisane gonadotrophins in the I.ezard (Anolis carolinensis). J. Endocr., 49, I 13 -124.

OTsukA S., 1956. On the extraction of the follicle stimulating and luteinizing substances of the Salmon. Endocrinol. Jap., 3, 272-277.

OTsukA S., I960. The similar substance to LH in mammalian Vertebrates. Acta. Endocr., 35, 1004I OIO.

Pappkoff H., Mahlmann L. J., Lr C. H., I967. Some chemical and physical properties of human ituitary follicle stimulating hormone. Biochemistry, 6, $3976,3982$.

Papkoff H., Licht P., I972. On the purification of turtle pituitary gonadotropin. Proc. Soc. Exp. Biol. Med., 139, 372-376.

Pickford G. E., Atz J. W., I957. The physiology of the pituitary gland of fishes. Acad. Press N. Y., ed.

Saxena B. B., Rathnam P., I97I. Chemical and immunochemical characteristics of the subunits of human pituitary LH and FSH. Proc. 2nd Int. Symp. Liège : Siructure activity relationships of protein and polypeptides hormone. Part. I, Excerpta Medica, I22-I3I.

Scanes C. G., Follet B. K., Goos H. J. Th., I972. Cross-reaction in a chicken LH-radioimmunoassay with plasma and pituitary extracts from various species. Gen. Comp. Endocrinol., 19, 596-600.

Sundararaj B. I., Goswami S. V., rg66. Effects of mammalian hypophysial hormones, placental. gonadotrophins, gonadal hormones, and adrenal cortico-steroids on ovulation and spawning in hypophysectomized Catfish Heteropneustes fossilis. J. Exp. Zool., 161, 287-296.

Sundararaj B. I., NAYYar S. K., I967. Effects of exogenous gonadotrophins and gonadal hormones on the testes and seminal vesicles of hypophysectomized Catfish Heteropneustes fossilis. Gen. Comp. Endocrinol., 8, 403-416.

Sundararaj B. I., Anand T. C., Donaldson E. W., I972. Effects of partially purified Salmon pituitary gonadotrophin on ovarian maintenance, ovulation and vitellogenesis in the hypophysectomized Catfish Heteropneustes fossilis (BLOCH.) Gen. Comp. Endocrinol, 18, Io2-II4-

Sundararaj B. I., Anand T. C., r972. Vertebrates reproduction. Part. II. Effects of piscine and mammalian gonadotropins on gametogenesis in the catfish Heteropneustes fossilis (BLOCH.). Gen. Comp. Endocrinol., suppl. 3, 688-702.

YamazaKi F., Donaldson E. M., rg68. The effects of partially purified Salmon pituitary gonadotropin on spermatogenesis vitellogenesis and ovulation in hypophysectomized Goldfish (Carassius auratus). Gen. Comp. Endocrinol., 11, 292-299. 